\title{
Comparative analysis of type 2 diabetes-associated SNP alleles identifies allele-specific DNA-binding proteins for the $K C N Q 1$ locus
}

\author{
MASAKI HIRAMOTO ${ }^{1,3}$, HARUHIDE UDAGAWA ${ }^{1}$, ATSUSHI WATANABE ${ }^{2}$, \\ KEISUKE MIYAZAWA ${ }^{3}$, NAOKO ISHIBASHI ${ }^{1}$, MIHO KAWAGUCHI ${ }^{1}$, TAKASHI UEBANSO ${ }^{1}$, \\ WATARU NISHIMURA ${ }^{1}$, TAKAO NAMMO ${ }^{1}$ and KAZUKI YASUDA ${ }^{1}$ \\ ${ }^{1}$ Department of Metabolic Disorder, Diabetes Research Center, Research Institute, \\ National Center for Global Health and Medicine, Tokyo 162-8655; ${ }^{2}$ Laboratory of Research Advancement, \\ Research Institute, National Center for Geriatrics and Gerontology, Obu, Aichi 474-8511; \\ ${ }^{3}$ Department of Biochemistry, Tokyo Medical University, Tokyo 160-8402, Japan
}

Received February 17, 2015; Accepted April 30, 2015

DOI: $10.3892 /$ ijmm.2015.2203

\begin{abstract}
Although recent genome-wide association studies (GWAS) have been extremely successful, it remains a big challenge to functionally annotate disease-associated single nucleotide polymorphisms (SNPs), as the majority of these SNPs are located in non-coding regions of the genome. In this study, we described a novel strategy for identifying the proteins that bind to the SNP-containing locus in an allele-specific manner and successfully applied this method to SNPs in the type 2 diabetes mellitus susceptibility gene, potassium voltage-gated channel, KQT-like subfamily Q, member 1 (KCNQ1). DNA fragments encompassing SNPs, and risk or non-risk alleles were immobilized onto the novel nanobeads and DNA-binding proteins were purified from the nuclear extracts of pancreatic $\beta$ cells using these DNA-immobilized nanobeads. Comparative analysis of the allele-specific DNA-binding proteins indicated that the affinities of several proteins for the examined SNPs differed
\end{abstract}

Correspondence to: Dr Kazuki Yasuda or Dr Masaki Hiramoto, Department of Metabolic Disorder, Diabetes Research Center, Research Institute, National Center for Global Health and Medicine, 1-21-1 Toyama, Shinjuku-ku, Tokyo 162-8655, Japan

E-mail: kyasuda@ri.ncgm.go.jp

E-mail: hiramoto@tokyo-med.ac.jp

Abbreviations: SNP, single nucleotide polymorphism; KCNQ1, potassium voltage-gated channel, KQT-like subfamily Q, member 1; NF-Y, nuclear transcription factor Y; CDKN1C, cyclin-dependent kinase inhibitor 1C; KCNQ1OT1, KCNQ1 opposite strand/antisense transcript 1; TRPM5, transient receptor potential cation channel, subfamily M, member 5; CUX1, cut-like homeobox 1

Key words: type 2 diabetes, $K C N Q 1$, single nucleotide polymorphism, DNA-binding proteins, affinity purification between the alleles. Nuclear transcription factor Y (NF-Y) specifically bound the non-risk allele of the SNP rs2074196 region and stimulated the transcriptional activity of an artificial promoter containing SNP rs2074196 in an allele-specific manner. These results suggest that SNP rs2074196 modulates the affinity of the locus for NF-Y and possibly induces subsequent changes in gene expression. The findings of this study indicate that our comparative method using novel nanobeads is effective for the identification of allele-specific DNA-binding proteins, which may provide important clues for the functional impact of disease-associated non-coding SNPs.

\section{Introduction}

Genome-wide association studies (GWAS) have identified numerous single nucleotide polymorphisms (SNPs) that are associated with human diseases, such as type 2 diabetes mellitus (1). However, the majority of these SNPs are located in non-coding regions of the genome, and the mechanisms through which they promote disease risk have not been completely elucidated. Potassium voltage-gated channel, KQT-like subfamily Q, member $1(K C N Q 1)$ is one of the genes most significantly associated with type 2 diabetes in different ethnic groups, particularly in East Asian populations, and type 2 diabetes-associated SNPs are also located mainly in an intron of $K C N Q 1$ (2-4). The $K C N Q 1$ gene encodes the $\alpha$ subunit of the voltage-gated potassium channel Kv7.1, and $K C N Q 1$ is also expressed in pancreatic $\beta$ cells, although the Kv7.1 channel is best characterized for its contribution to cardiac function (5). A recent study indicated that increased KCNQ1 protein expression limits insulin secretion from pancreatic $\beta$ cells by regulating the potassium channel current (6). However, to date, and to the very best of our knowledge, no data have been presented regarding the association between the disease-associated variants and the expression of $K C N Q 1$.

Recent studies from the ENCODE project have confirmed that disease-associated variants are enriched in regulatory 
DNA and that promoters and distal elements engage in multiple long-range interactions to form complex networks (7-10). Therefore, these SNPs in the KCNQ1 gene may affect the expression of nearby or long-distance genes. Among the candidate genes close to $K C N Q 1$, the loss of cyclin-dependent kinase inhibitor $1 \mathrm{C}(C D K N 1 C)$ in focal lesions in infants with hyperinsulinism has been shown to correlate with the increased proliferation of pancreatic $\beta$ cells (11), and targeting CDKN1C promotes adult human $\beta$ cell replication (12). Furthermore, the long non-coding RNA, KCNQ1 opposite strand/antisense transcript 1 (KCNQ1OT1), regulates the expression of CDKN1C through imprinting (13-15). Transient receptor potential cation channel, subfamily $\mathrm{M}$, member 5 (TRPM5), another nearby gene, has been reported to regulate glucose-stimulated insulin secretion in pancreatic $\beta$ cells (16-19). Again, however, to the best of our knowledge, no data to date have been presented regarding the association between the disease-associated variants and the expression of these nearby genes.

In order to analyze the molecular mechanisms linking these non-coding variants with type 2 diabetes mellitus, it is crucial to identify DNA-binding proteins that recognize the nucleotide sequence surrounding disease-associated variants in an allele-specific manner; however, to the best of our knowledge, there have only been a few reports that have been successful in the identificatoin of such proteins. We and our colleagues have previously developed novel nanobeads composed of glycidyl methacrylate and styrene, and demonstrated that these nanobeads enable the rapid and efficient purification of 'bait' binding proteins, including transcription factors using DNA fragments as bait (20-22). In the present study, we applied further-improved magnetic nanobeads (23), and aimed to identify the proteins that bind the $K C N Q 1$ intronic locus containing disease-associated SNPs in an allele-specific manner. We also investigated the function of these proteins in order to clarify the association between genetic variants and the risk of type 2 diabetes.

\section{Materials and methods}

Cells. The rat pancreatic $\beta$-cell line, INS-1 (24), which had kindly been provided by Dr C.B. Wollheim and Dr N. Sekine (University of Geneva, Geneva, Switzerland), was cultured at $37^{\circ} \mathrm{C}$ in a $5 \% \mathrm{CO}_{2}$ atmosphere in RPMI-1640 medium which was supplemented with $10 \%$ fetal bovine serum, $10 \mathrm{mM}$ HEPES, $1 \mathrm{mM}$ sodium pyruvate and $50 \mathrm{mM}$ 2-mercaptoethanol. KP-3 (human pancreatic cancer), KP-4 (pancreatic ductal cell carcinoma), QGP-1 (human pancreatic endocrine) and SUIT-2 (human pancreatic cancer) cells were obtained from the Health Science Research Resources Bank (Osaka, Japan). AsPC-1 (human pancreatic adenocarcinoma), Capan-1 (human pancreatic ductal adenocarcinoma), 293T and HeLa cells were obtained from the American Type Culture Collection (ATCC, Manassas, VA, USA). The KP-3, QGP-1, SUIT-2 and AsPC-1 cells were cultured in RPMI-1640 medium which was supplemented with $10 \%$ fetal bovine serum. The KP-4 and Capan-1 cells were cultured in Iscove's modified Dulbecco's medium (IMDM) supplemented with $10 \%$ fetal bovine serum. The 293T and HeLa cells were cultured in Dulbecco's modified Eagle's medium (DMEM) which was supplemented with $10 \%$ fetal bovine serum.
Preparation of DNA-immobilized nanobeads. The oligonucleotides used in the present study were synthesized at Japan BioServices Co. Ltd. (Saitama, Japan). These oligonucleotides contained either risk or non-risk alleles through single-nucleotide substitution (Table I). DNA-immobilized nanobeads were prepared as previously described with slight modifications $(21,25)$. Two complementary oligonucleotides were phosphorylated, annealed and then ligated to produce oligomers that ranged mainly from 100 to 3,000 bp. These oligomers containing tandemly repeated SNP regions were applied to the NICK Column according to the manufacturer's instructions (GE Healthcare, Buckinghamshire, UK). The second fractions of the NICK Column were ready for coupling onto FG beads (Tamagawa Seiki Co., Ltd., Nagano, Japan) (23). One milligram of FG beads was then mixed with $100 \mu \mathrm{g}$ of prepared DNA, and the coupling reaction was carried out at $50^{\circ} \mathrm{C}$ for $24 \mathrm{~h}$.

Affinity purification. Nuclear extracts were prepared from the INS-1 cells according to the method described in the study by Dignam et al (26). DNA-immobilized nanobeads $(200 \mu \mathrm{g})$ were equilibrated with binding buffer $(20 \mathrm{mM}$ HEPES-NaOH, $\mathrm{pH} 7.9,10 \%$ glycerol, $100 \mathrm{mM} \mathrm{KCl}, 1 \mathrm{mM} \mathrm{MgCl}_{2}, 0.2 \mathrm{mMCaCl}_{2}$, $0.2 \mathrm{mM}$ EDTA, $1 \mathrm{mM}$ DTT and $0.2 \mathrm{mM}$ PMSF). INS-1 nuclear extracts $(200 \mu \mathrm{g})$ were mixed with $100 \mu \mathrm{g}$ of single-stranded DNA (Ambion/Life Technologies, Austin, TX, USA) and $10 \mu \mathrm{g}$ of poly(dI-dC):poly(dI-dC) (Sigma-Aldrich, St. Louis, MO, USA), and then incubated with equilibrated DNA-immobilized nanobeads for $4 \mathrm{~h}$ at $4^{\circ} \mathrm{C}$ using a RT-50 rotator (Taitec Corp., Saitama, Japan). After washing with binding buffer 3 times, the bound proteins were eluted with binding buffer supplemented with $1 \mathrm{M} \mathrm{KCl}$ (final concentration).

Mass spectrometry. Affinity-purified proteins were separated using SDS-PAGE and subjected to silver staining as described in the study by Shevchenko et al (27). The specific protein bands were excised and mass spectrometry was then performed as previously described (28). Band slices were reduced with $10 \mathrm{mM}$ dithiothreitol, alkylated with $55 \mathrm{mM}$ iodoacetamide and digested with sequencing grade modified trypsin $(12 \mu \mathrm{g} / \mathrm{ml}$; Promega Corp., Madison, WI, USA) overnight. The extracted peptides were desalted with ZipTip C18 (Millipore, Billerica, MA, USA) and separated by nano-flow liquid chromatography (LC) (Paradigm MS4; Michrom BioResources, Inc., Auburn, CA, USA) using a reverse phase C18 column (Magic C18, $0.2 \times 50 \mathrm{~mm}$; Michrom BioResources, Inc.). The LC eluent was coupled to a micro-ionspray source attached to an LCQ Advantage MAX mass spectrometer (Thermo Fisher Scientific Inc., Waltham, MA, USA). All MS/MS spectra were searched using the TurboSEQUEST algorithm within the BioWorks 3.2 software (Thermo Fisher Scientific Inc.).

Immunoblot analysis. Nuclear extracts and affinity-purified fractions were fractionated by SDS-PAGE and electrotransferred onto a polyvinylidene difluoride membrane (Millipore). The membrane was incubated with 5\% non-fat milk for $1 \mathrm{~h}$ and incubated with antibody against nuclear transcription factor Y, beta (NF-YB) overnight (sc-13045; Santa Cruz Biotechnology, Inc., Santa Cruz, CA, USA), followed by incubation with peroxidase-conjugated anti-rabbit IgG (no. 7074; Cell Signaling Technology, Inc., Danvers, MA, USA) for $1 \mathrm{~h}$. 
Table I. Oligonucleotides used for the preparation of the DNA-immobilized nanobeads.

\begin{tabular}{|c|c|c|c|}
\hline SNP & Allele & & Primer sequence $\left(5^{\prime} \rightarrow 3^{\prime}\right)$ \\
\hline \multirow[t]{4}{*}{ rs2237892 } & Risk & Forward & GGGGGTTTGCCACC[C]GGGGTGAGG \\
\hline & & Reverse & СССССССТСАСССС[G]GGTGGCAAA \\
\hline & Non-risk & Forward & GGGGGTTTGCCACC[T]GGGGTGAGG \\
\hline & & Reverse & 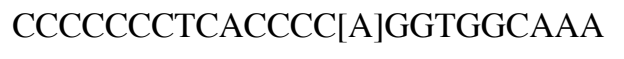 \\
\hline \multirow[t]{4}{*}{ rs2237895 } & Risk & Forward & GGGGGAGTGGTCCC[C]GGGGTCGGG \\
\hline & & Reverse & CCCCCCCCGACCCC $[\mathrm{G}] \mathrm{GGGACCACT}$ \\
\hline & Non-risk & Forward & GGGGGAGTGGTCCC[A]GGGGTCGGG \\
\hline & & Reverse & CCCCCCCCGACCCC[T]GGGACCACT \\
\hline \multirow[t]{4}{*}{ rs 151290} & Risk & Forward & GGGGGTGAGCCCAG[C]CCCCTGGGC \\
\hline & & Reverse & CCCCCGCCCAGGGG[G]CTGGGCTCA \\
\hline & Non-risk & Forward & GGGGGTGAGCCCAG[A]CCCCTGGGC \\
\hline & & Reverse & CCCCCGCCCAGGGG[T]CTGGGCTCA \\
\hline \multirow[t]{4}{*}{ rs2074196 } & Risk & Forward & GGGGGGGGGCTTCA[G]TGGAGCCCG \\
\hline & & Reverse & CCCCCCGGGCTCCA[C]TGAAGCCCC \\
\hline & Non-risk & Forward & GGGGGGGGGCTTCA[T]TGGAGCCCG \\
\hline & & Reverse & CCCCCCGGGCTCCA[A]TGAAGCCCC \\
\hline \multirow[t]{4}{*}{ rs2283228 } & Risk & Forward & GGGGGGCTGAAAGC[A]CTGGTTAAA \\
\hline & & Reverse & CCСССТTTAACCAG[T]GCTTTCAGC \\
\hline & Non-risk & Forward & GGGGGGCTGAAAGC[C]CTGGTTAAA \\
\hline & & Reverse & СССССТTТАAСCAG[G]GCTTTCAGC \\
\hline \multirow[t]{4}{*}{ rs2237897 } & Risk & Forward & GGGGGAGCTGGGGA[C]GAGGGGCCT \\
\hline & & Reverse & CCCCCAGGCCCCTC[G]TCCCCAGCT \\
\hline & Non-risk & Forward & GGGGGAGCTGGGGA[T]GAGGGGCCT \\
\hline & & Reverse & CCCCCAGGCCCCTC[A]TCCCCAGCT \\
\hline
\end{tabular}

Parentheses indicate the positions of SNPs. SNP, single nucleotide polymorphism.

Visualization was accomplished using enhanced chemiluminescense reagents (Millipore) and analyzed using the ChemiDoc XRS+ imaging system (Bio-Rad, Hercules, CA, USA).

Preparation of radiolabeled recombinant proteins. Complementary DNA (cDNA) for nuclear transcription factor $\mathrm{Y}$, alpha $(N F-Y A), N F-Y B$, nuclear transcription factor Y, gamma $(N F-Y C)$ and cut-like homeobox $1(C U X I)$ were amplified by RT-PCR from the total RNA prepared from HeLa cells, inserted into the pUC119 vector, and sequenced using an ABI PRISM 3700 sequence analyzer (Applied Biosystems, Foster City, CA, USA). The preparation of radiolabeled recombinant proteins was then performed as previously described (28). T7 promoter-tagged DNA fragments of these cDNAs were amplified using PCR with two primers: T7 promoter fused to a 5' sequence of each cDNA and polyT fused to a 3' complementary sequence of each cDNA. These amplified DNA fragments were used to synthesize 35S-radiolabeled recombinant proteins in a coupled transcription/translation system according to the protocol of the manufacturer (Promega Corp.).

Binding assay. DNA-immobilized nanobeads or control nanobeads $(200 \mu \mathrm{g})$ were equilibrated with binding buffer as described above and incubated with $200 \mu \mathrm{l}$ of the radiolabeled proteins (NF-YA, NF-YB, NF-YC and CUX1) at $4^{\circ} \mathrm{C}$ for $4 \mathrm{~h}$ using a RT-50 rotator. After washing with binding buffer, the bound proteins were eluted by boiling for $5 \mathrm{~min}$ with SDS-PAGE sample buffer. The eluates and inputs were subjected to SDS-PAGE. The gels were dried, and autoradiography was then performed to visualize the radiolabeled proteins.

Construction of plasmids. Reporter plasmids that contain the luciferase gene under the control of the SNP rs2074196 region were constructed as follows: complementary oligonucleotides containing 3 copies of the SNP region were synthesized. These oligonucleotides were annealed and cloned into the pGL4.10 vector (Promega Corp.) along with the thymidine kinase promoter derived from pGL4.74 (Promega Corp.).

To construct mammalian expression plasmids, cDNA of $N F-Y A, N F-Y B, N F-Y C$ and $C U X 1$ were amplified by PCR and subcloned into the pcDNA6 vector (Invitrogen Life Technologies, Carlsbad, CA, USA) to allow the expression of these cDNAs under the control of a CMV promoter. During PCR amplification, FLAG-tag was fused at the C-terminus of NF-YA; HA-tag was fused at the C-terminus of NF-YB; and Myc-tag was fused at the C-terminus of NF-YC. All the 
A

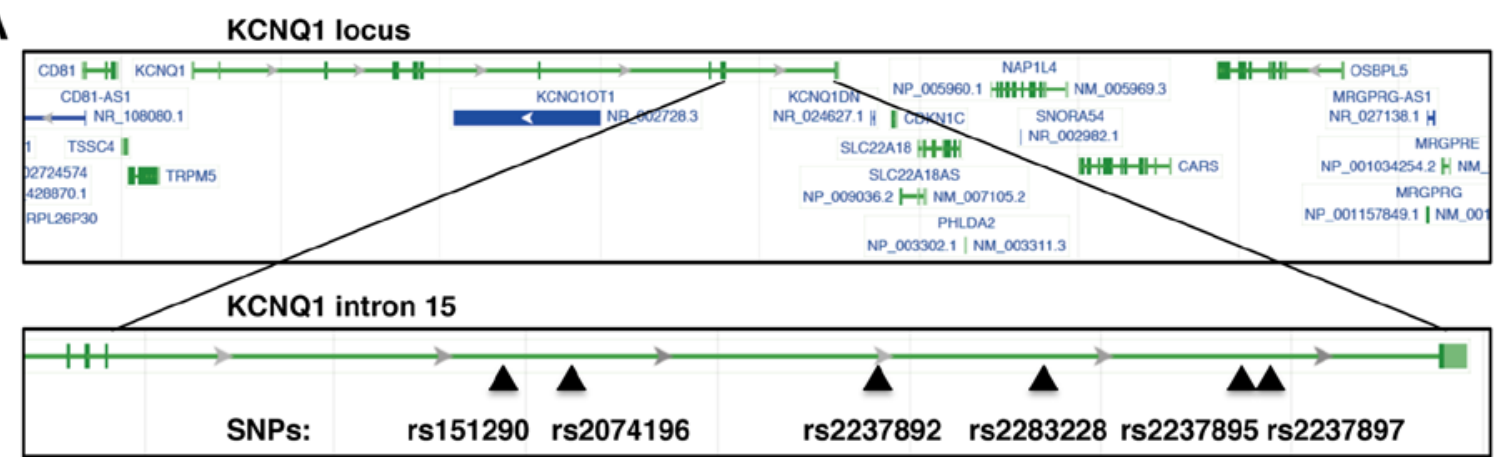

B

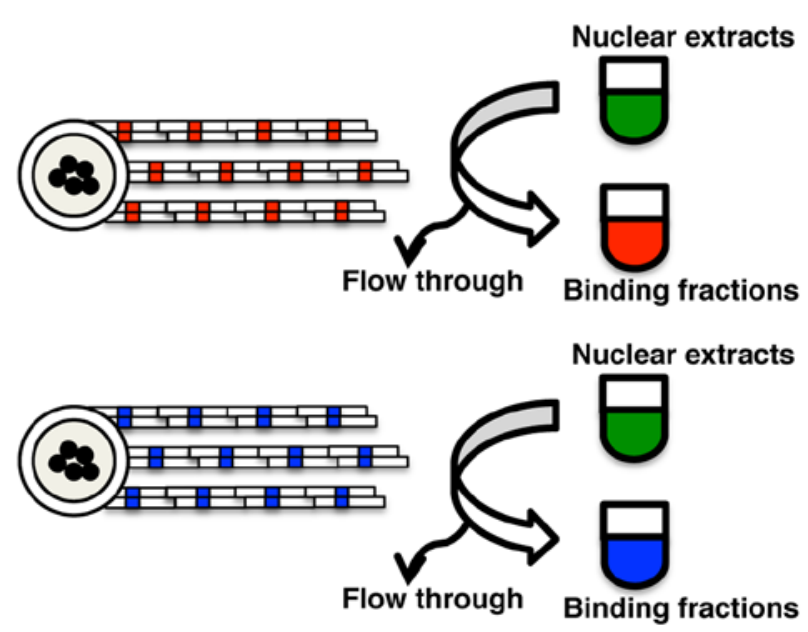

\begin{abstract}
Oligo Risk-F Oligo Risk-R
\end{abstract}

Oligo NR-F Oligo NR-R

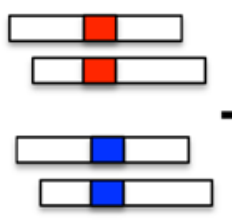

FG beads

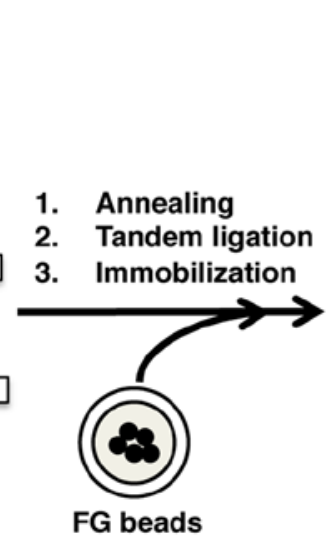

Nuclear extracts

Binding fractions

Figure 1. Schematic experimental design. (A) Screen shot of the NCBI browser showing the human potassium voltage-gated channel, KQT-like subfamily Q, member 1 (KCNQ1) gene locus. Positions of the single nucleotide polymorphisms (SNPs) examined in this study are indicated by arrows. (B) Workflow for preparing of DNA-immobilized nanobeads and purifying of allele-specific binding proteins.

sequences were confirmed using an ABI PRISM 3700 sequence analyzer.

Luciferase assay. One of the constructed luciferase reporter plasmids (100 ng) and the control Renilla luciferase reporter vector pGL4.74 (10 ng) were co-transfected into the 293T cells ( $2 \times 10^{5}$ cells) along with mammalian expression vectors: NF-Ys (NF-YA-FLAG, NF-YB-HA, and NF-YC-Myc, $300 \mathrm{ng}$ each), CUX1 (900 ng), or empty pcDNA6 (900 ng) using Lipofectamine 2000 (Invitrogen Life Technologies) and seeded into a 24-well plate. Twelve hours after transfection, the medium was changed. Forty-eight hours after transfection, cell extracts were prepared, and Firefly luciferase activities and Renilla luciferase activities were determined using a Dual-Glo Luciferase assay system (Promega Corp.) and a CentroPRO LB 962 Microplate Luminometer (Berthold Technologies, Bad Wildbad, Germany).

Chromatin immunoprecipitation assay. The preparation of the samples was performed using a Magna ChIP G kit according to the manufacturer's instructions (Millipore, Temecula, CA, USA). The samples were sonicated for 10 rounds of 30 pulses each ( $0.5 \mathrm{sec}$ on and $0.5 \mathrm{sec}$ off) using a Branson Sonifier S-450 digital ultrasonic cell disruptor/homogenizer (Branson Ultrasonics Corp., Danbury, CT, USA) at $15 \%$ amplitude. The efficiency of sonication was assessed to ensure that the majority of chromatin fragments were in the range of 200-1,000 bp. The sonicated samples were immunoprecipitated overnight at $4^{\circ} \mathrm{C}$ with $2.5 \mu \mathrm{g}$ of anti-Myc-tag antibody (no. 2276; Cell Signaling Technology, Inc.) or normal mouse IgG (Millipore). The immunoprecipitated DNA was quantified by qPCR performed on the input and bound fractions, using the Fast SYBR-Green Master Mix and a StepOne Real-Time PCR system (both from Applied Biosystems). The primers used for qPCR were as follows: 5'-GAGCCAGTTGTTCCCA AACC-3' and 5'-TAGGCTTGTGTCCCCAGTCC-3' for SNP rs2074196, and 5'-CGCAAAAGGAGGGGAGAG-3' and 5'-GCCGCTGGGTTTTATAGGG-3' for the ACTB promoter.

Statistical analysis. All data are expressed as the means \pm SD. Statistical analysis was performed using an unpaired two-tailed Student's t-test. A value of $\mathrm{P}<0.01$ was considered to indicate a statistically significant difference.

\section{Results}

Purification of allele-specific DNA-binding proteins by novel magnetic nanobeads. In this study, we aimed to identify proteins that bind the genomic region encompassing the type 2 diabetes-associated SNPs: rs151290, rs2074196, rs2237892, rs2283228, rs2237895 and rs2237897 (Fig. 1A). In order to identify the allele-specific binding proteins, we first prepared 

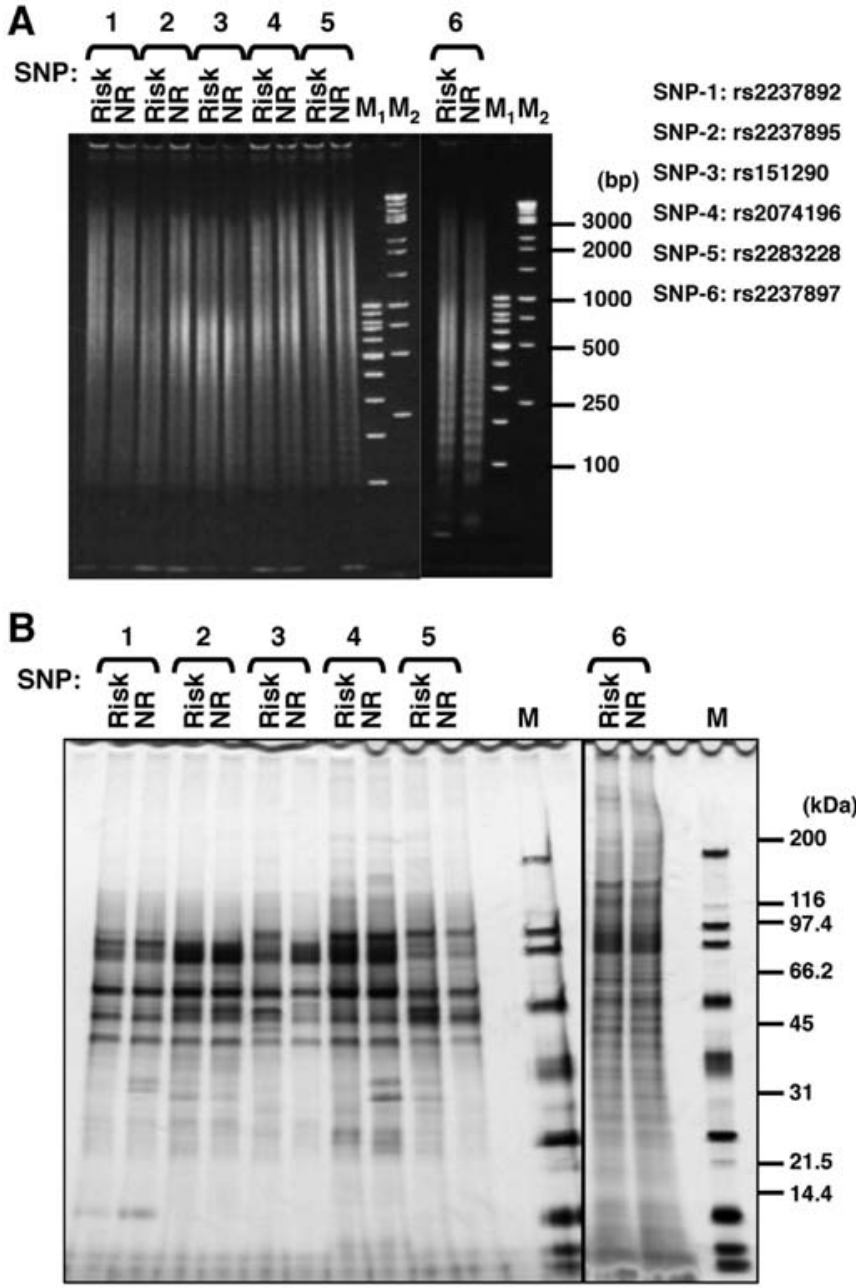

Figure 2. Purification of allele-specific binding proteins. (A) Preparation of immobilizing DNA. Tandemly ligated DNAs were separated using acrylamide gel electrophoresis (5-20\% gradient gel) and visualized using ethidium bromide staining. (B) Aliquots of purified fractions were separated using SDS-PAGE (5-20\% gradient gel) and visualized using silver staining.

2 types of beads, risk and non-risk allele beads, by immobilizing each allelic DNA onto magnetic nanobeads (Table I and Fig. 1B). The lengths of the immobilized DNA fragments were almost the same (100-3,000 bp) for the risk and non-risk alleles (Fig. 2A). Using these nanobeads, we purified DNA-binding proteins from the nuclear extract of INS-1 pancreatic $\beta$ cells and analyzed the bound proteins using SDS-PAGE (Fig. 2B). We found that the affinities of several proteins for each examined SNP differed between alleles. There were obvious allele-specific bands, particularly in the SNP rs151290 and SNP rs2074196 regions. We decided to perform the following analysis with SNP rs2074196, as this was among the 3 SNPs (rs2074196, rs2237892 and rs2237895) which showed the best association P-values with type 2 diabetes in our previous study (2).

Identification of allele-specific DNA-binding proteins. We compared the bound proteins of the risk and non-risk alleles and selected 4 differentially bound protein bands (Fig. 3A). Following mass spectrometry, we identified 3 proteins, CUX1, NF-YC and NF-YA. One protein band was not determined using mass spectrometry. However, we speculated that the band
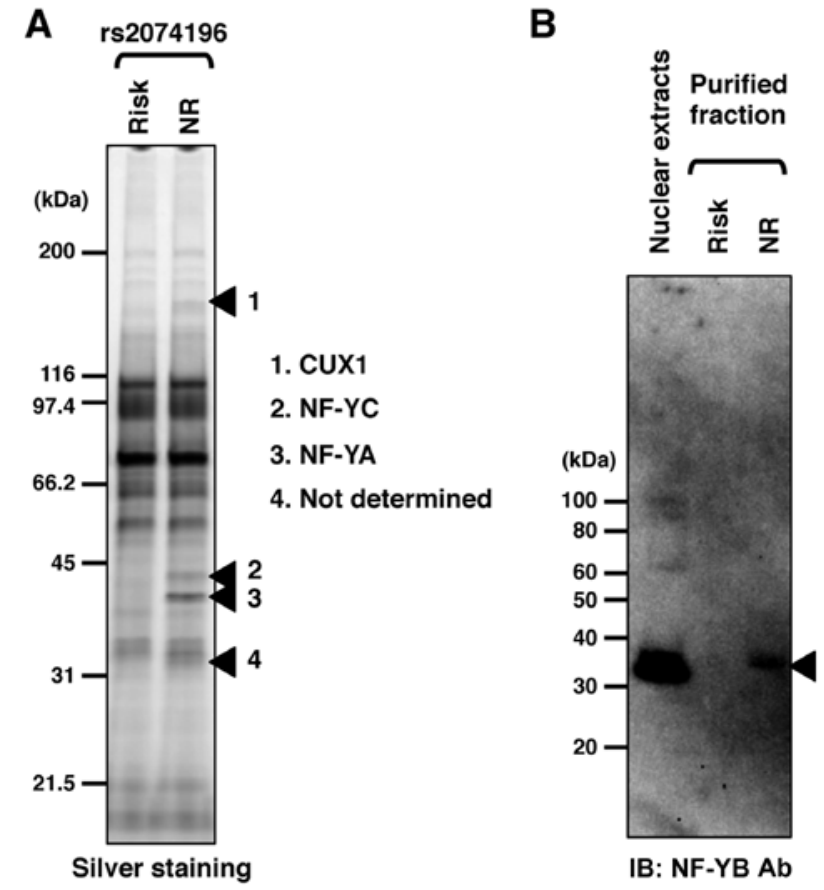

Figure 3. Identification of allele-specific binding proteins for single nucleotide polymorphism (SNP) rs2074196. (A) The 4 polypeptides indicated by arrows were analyzed using ion-spray mass spectrometry. (B) INS-1 cell nuclear extracts and purified fractions were subjected to immunoblot analysis (IB) using a specific antibody against nuclear transcription factor Y, beta (NF-YB).

was NF-YB, due to the size of the protein band and the lack of a partner protein for the NF-Y ternary complex. Immunoblot analysis using a specific antibody against NF-YB confirmed that NF-YB had been purified using DNA-immobilized nanobeads in an allele-specific manner (Fig. 3B).

Evaluation of allele-specific DNA-binding proteins. We then confirmed the DNA-binding activities of NF-Y and CUX1 in vitro using recombinant proteins. NF-YA, NF-YB or NF-YC alone exhibited no binding activity or only non-specific binding activity against the genomic region encompassing SNP rs2074196 (Fig. 4A). The ternary complex of NF-YA, NF-YB and NF-YC exhibited specific binding activity against the non-risk allele of the genomic region encompassing SNP rs2074196 (Fig. 4B). CUX1 also exhibited specific binding activity against the non-risk allele of the genomic region encompassing SNP rs2074196 (Fig. 4B). These DNA-binding specificities against the non-risk allele of recombinant NF-Y and CUX1 were consistent with those observed in the silver staining of purified proteins from the INS-1 cell nuclear extracts (Figs. 2B and 3A).

SNP rs2074196 modulates NF-Y-dependent transcriptional activity. We then examined the transcriptional activities of NF-Y and CUX1 using luciferase reporter assay. As illustrated in Fig. 5A, we prepared 4 reporter constructs: risk allele and forward orientation, non-risk allele and forward orientation, risk allele and reverse orientation, and non-risk allele and reverse orientation. The basal luciferase activities, which are mediated by endogenous transcription factors possibly including NF-Y and CUX1, differed among the constructs (Fig. 5B): 
A
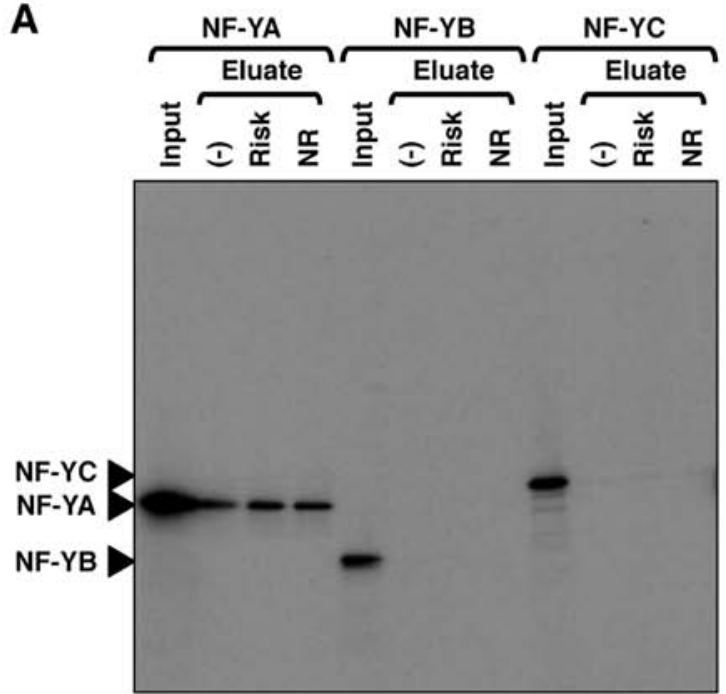

Autoradiography

B

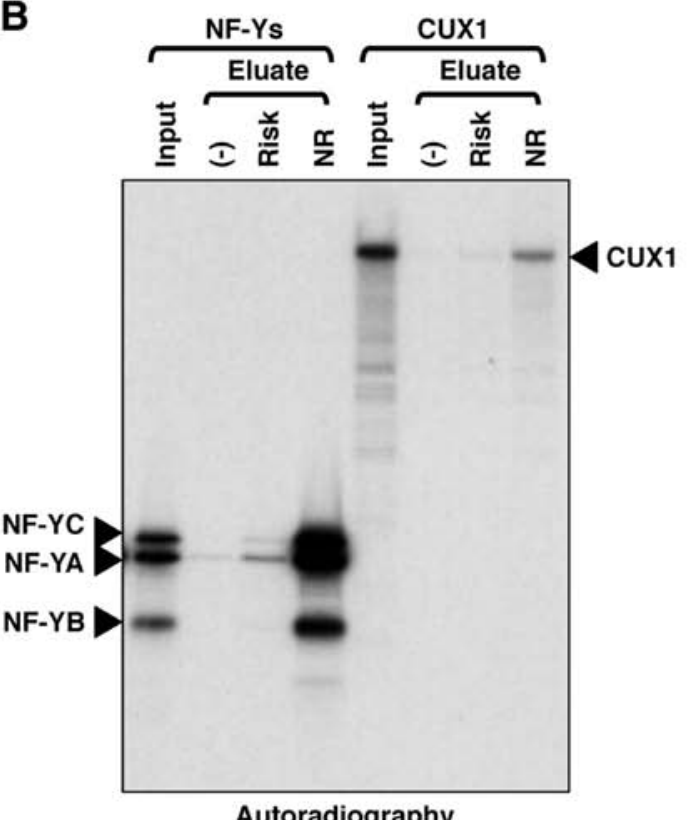

Figure 4. Evaluation of binding activity of identified proteins. (A) In vitro radiolabeled nuclear transcription factor Y, alpha (NF-YA), beta (NF-YB), or gamma (NF-YC) was separately mixed with DNA-immobilized nanobeads of the single nucleotide polymorphism (SNP) rs2074196 region (risk or non-risk) or negative control nanobeads without DNA (-). Ten percent of input and eluate fractions were separated using SDS-PAGE and visualized using autoradiography. (B) In vitro radiolabeled nuclear transcription factor Y (NF-Y) ternary complex (NF-YA/NF-YB/NF-YC) or cut-like homeobox 1 (CUX1) were mixed with DNA-immobilized nanobeads of the SNP rs2074196 region (risk or non-risk) or negative control nanobeads without DNA (-). Ten percent of input and eluate fractions were separated using SDS-PAGE and visualized using autoradiography.

non-risk-forward, 1.60-fold; risk-reverse, 0.57-fold; and non-risk-reverse, 0.84 -fold higher compared to risk-forward. Hence, the basal luciferase activities of non-risk-forward were the highest for these 4 constructions in 293T cells.

When the expression constructs of NF-YA, NF-YB and NF-YC were co-transfected, the luciferase activities of each reporter construct were upregulated (Fig. 5B): risk-forward, 1.16-fold; non-risk-forward, 3.45-fold, risk-reverse, 1.07-fold; and non-risk-reverse, 1.66 -fold compared to basal risk-rorward;
A

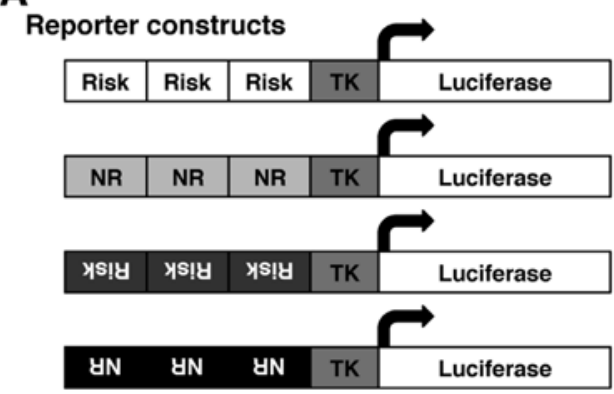

B

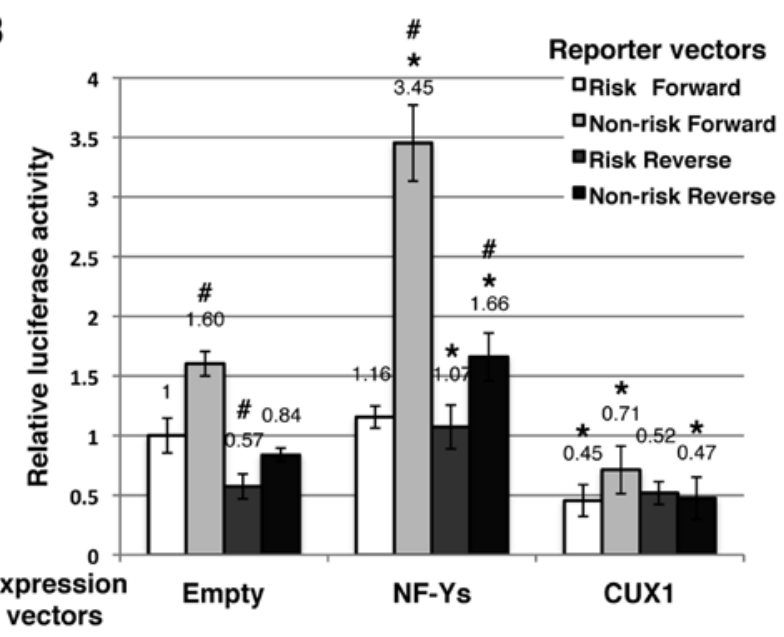

Figure 5. Effects of nuclear transcription factor Y (NF-Y) or cut-like homeobox 1 (CUX1) on transcriptional activity under the control of the genomic region encompassing single nucleotide polymorphism (SNP) rs2074196. (A) Construction of 4 types of Firefly luciferase reporter vectors. (B) $293 \mathrm{~T}$ cells were transfected withone of each firefly luciferase reporterconstructs and control Renilla luciferase reporter vector pGL4.74 along with the mammalian expression vectors, NF-Ys [nuclear transcription factor Y, alpha (NF-YA)-FLAG, nuclear transcription factor $\mathrm{Y}$, beta (NF-YB)-HA, and nuclear transcription factor Y, gamma (NF-YC)-Myc], CUX1, or empty. Firefly luminescence was normalized using renilla luminescence. Data are the means \pm SD calculated from 4 independent replicates. Statistical significance: ${ }^{*} \mathrm{P}<0.01$, relative to the empty expression vector in each reporter vector; ${ }^{\#} \mathrm{P}<0.01$, relative to the risk-forward reporter vector in each expression vector calculated by an unpaired Student's t-test, two-tailed.

in addition non-risk-forward, 2.16-fold, risk-reverse, 1.87-fold, and non-risk-reverse, 1.98 -fold compared to the basal value of each construction. These results confirmed that the NF-Y complex upregulated the transcription under the control of the genomic region encompassing SNP rs2074196, particularly for the non-risk allele and forward orientation.

When the expression construct of CUX1 was co-transfected, the luciferase activities of each reporter construct were downregulated (Fig. 5B). These results indicated that CUX1 downregulated the transcription under the control of the genomic region encompassing SNP rs2074196; however, no allele specificity was observed.

SNP rs2074196 modulates the affinity of the locus for NF-Y. We further examined the DNA-binding activities of NF-Y in cell lines using chromatin immunoprecipitation assay using 6 cell lines: QGP-1, SUIT-2, KP-4, KP-3, AsPC-1 and Capan-1. The QGP-1 and SUIT-2 cells were found to be homozygous for the non-risk allele, the KP-4 and KP-3 were heterozygous 

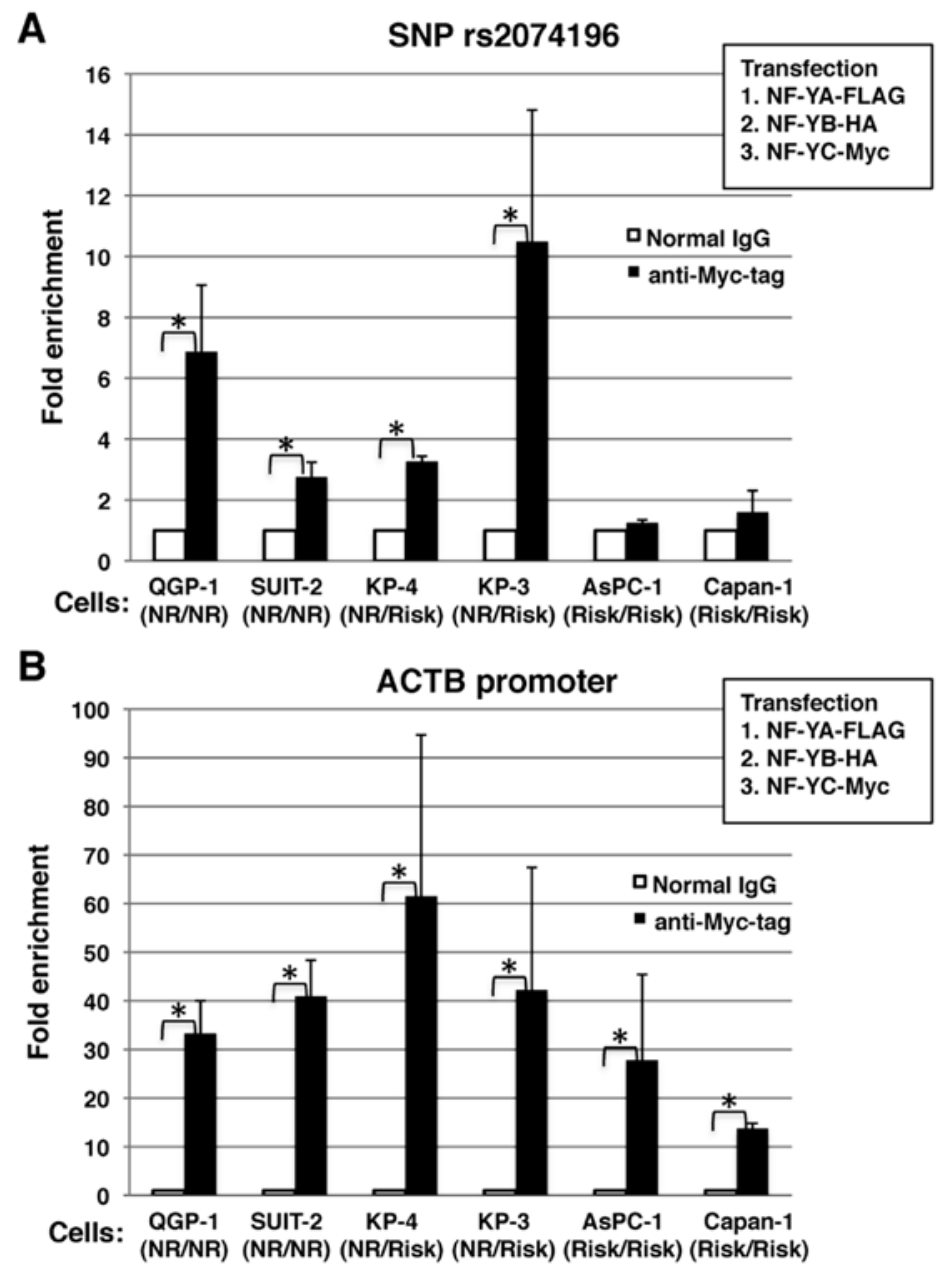

Figure 6. Allele-specific binding activity of nuclear transcription factor Y (NF-Y) for the genomic region encompassing single nucleotide polymorphism (SNP) s2074196. (A) Indicated cell lines were transfected with expression vectors for nuclear transcription factor Y, alpha (NF-YA)-FLAG, nuclear transcription factor Y, beta (NF-YB)-HA, and nuclear transcription factor Y, gamma (NF-YC)-Myc together. DNA-protein complexes were immunoprecipitated with anti-Myc-tag antibody or normal mouse IgG. Enrichment of the target genomic region encompassing SNP rs2074196 was estimated using qPCR, and fold enrichment anti-Myc-tag antibody vs. normal mouse IgG is displayed. (B) Binding activity of NF-Y for the ACTB promoter. Enrichment of the target ACTB promoter region was estimated using qPCR, and fold enrichment anti-Myc-tag antibody vs. normal mouse IgG is displayed. "P<0.01, unpaired Student's $\mathrm{t}$-test, two-tailed. All data are expressed as the means $\pm \mathrm{SD}$ from 4 independent experiments.

for the risk and non-risk allele, and the AsPC-1 and Capan-1 cells were homozygous for the risk allele. We transfected these cells with the expression vectors, NF-YA-FLAG, NF-YB-HA, and NF-YC-Myc. As illustrated in Fig. 6A, the genomic region encompassing SNP rs2074196 was enriched in the QGP-1, SUIT-2, KP-4 and KP-3 cells, but not in the AsPC-1 and Capan-1 cells. As a positive control, the genomic region of the ACTB promoter containing NF-Y-binding CCAAT-box elements was enriched in all the examined cell lines (Fig. 6B). These results confirmed that NF-Y specifically bound the non-risk allele of the endogenous $K C N Q 1$ intronic region encompassing SNP rs2074196.

\section{Discussion}

GWAS have indicated that SNPs in the KCNQ1 locus are associated with type 2 diabetes (2-4). Since the majority of these SNPs are located in the intronic region of the KCNQ1 gene and are associated with an impaired insulin secretion, we hypothesized that some of these SNP-containing regions may participate in regulating gene expression in pancreatic $\beta$ cells as cis-regulatory elements, such as enhancers and that SNPs may affect such activity of this locus.

Novel nanobeads, as we and our colleague have previously reported $(20-23,25,28)$, have been proven to be useful for screening proteins that bind to a variety of baits. Since one of the major features of the beads was a very low background, we hypothesized that they may be also applied to purify DNA-binding proteins in an allele-specific manner. Indeed, we successfully found that the affinities of several proteins for the examined SNP regions in the $K C N Q 1$ gene locus differed between alleles. NF-Y specifically bound the non-risk allele of the SNP rs2074196 region and stimulated the transcriptional activity of the artificial promoter containing SNP rs2074196 in an allele-specific manner. These results suggest that SNP rs2074196 modulates the affinity of the locus for NF-Y and possibly induces subsequent changes in gene expression, which may provide important clues as to how type 2 diabetes-associated SNPs in the KCNQ1 gene locus promote disease risk. 
Recent studies have confirmed that NF-Y is necessary for hematopoietic stem cell proliferation and survival. $N F-Y A$ deletion creates an accumulation of hematopoietic stem cells in the $\mathrm{G} 2 / \mathrm{M}$ phase, accompanied by the dysregulation of multiple genes that influence cell cycle control and apoptosis (29). Another group reported that the inactivation of $N F-Y A$ in postnatal liver causes hepatocellular degeneration, lipid deposition and endoplasmic reticulum stress (30), indicating that NF-Y is a key transcription factor controlling endoplasmic reticulum function and metabolic processes in mature hepatocytes. Although the function of NF-Y in pancreatic $\beta$ cells has not been well studied, these observations suggest that NF-Y participates in the regulation of $\beta$ cell development and function. Future identification of the target genes regulated by this locus encompassing SNP rs2074196 and NF-Y are required to provide further information.

In addition, we cannot exclude the possibility that other genomic regions harboring intronic SNPs in the $K C N Q 1$ gene may also be functional and contribute to susceptibility to type 2 diabetes as gene expression may be regulated by several independent or correlated regions containing respective SNPs. Although SNP rs2074196 is the only variant extensively examined thus far, we have already detected allele-specific binding proteins for other SNP regions (Fig. 2B). Further experiments on each allele-specific binding protein and the interaction between these SNPs should clarify the molecular association between SNPs in the KCNQ1 locus and susceptibility to type 2 diabetes.

Although recent GWAS have been extremely successful (1-4), it remains a big challenge to functionally annotate SNPs in susceptibility genes that are very often localized in non-coding regions. For this purpose, it is essential to identify DNA-binding proteins that recognize the nucleotide sequence surrounding disease-associated variants. Our comparative analysis between alleles using DNA-immobilized nanobeads enabled systematic identification of allele-specific DNA-binding proteins in multiple SNP regions. We believe that this system is also very useful for investigating the molecular mechanisms linking non-coding genetic variants to the risk of common diseases in general.

\section{Acknowledgements}

We would like to thank Mr. Dai Suzuki and Ms. Kazuko Nagase for providing technical assistance. This study was supported by grants from the Japan Society for the Promotion of Science (JSPS) Grants-in-Aid for Scientific Research (KAKENHI), grant no. 22510216 (to M.H.), the Japan Diabetes Foundation (to M.H.), the Leading Research Project of Ministry of Education, Culture, Sports, Science and Technology, Japan (to K.Y.), National Institute of Biomedical Innovation (to K.Y.) and National Center for Global Health and Medicine (to K.Y.).

\section{References}

1. Imamura $M$ and Maeda S: Genetics of type 2 diabetes: The GWAS era and future perspectives (Review). Endocr J 58: 723-739, 2011.

2. Yasuda K, Miyake K, Horikawa Y, Hara K, Osawa H, Furuta H, Hirota Y, Mori H, Jonsson A, Sato Y, et al: Variants in KCNQ1 are associated with susceptibility to type 2 diabetes mellitus. Nat Genet 40: 1092-1097, 2008
3. Unoki H, Takahashi A, Kawaguchi T, Hara K, Horikoshi M, Andersen G, Ng DP, Holmkvist J, Borch-Johnsen K, Jørgensen T, et al: SNPs in KCNQ1 are associated with susceptibility to type 2 diabetes in East Asian and European populations. Nat Genet 40: 1098-1102, 2008.

4. Voight BF, Scott LJ, Steinthorsdottir V, Morris AP, Dina C, Welch RP,Zeggini E, Huth C, Aulchenko YS, Thorleifsson G, et al; MAGIC investigators; GIANT Consortium: Twelve type 2 diabetes susceptibility loci identified through large-scale association analysis. Nat Genet 42: 579-589, 2010.

5. Neyroud N, Tesson F, Denjoy I, Leibovici M, Donger C, Barhanin J, Fauré S, Gary F, Coumel P, Petit C, et al: A novel mutation in the potassium channel gene KVLQT1 causes the Jervell and Lange-Nielsen cardioauditory syndrome. Nat Genet 15: 186-189, 1997.

6. Yamagata K, Senokuchi T, Lu M, Takemoto M, Fazlul Karim M, Go C, Sato Y, Hatta M, Yoshizawa T, Araki E, et al: Voltage-gated $\mathrm{K}^{+}$channel KCNQ1 regulates insulin secretion in MIN6 $\beta$-cell line. Biochem Biophys Res Commun 407: 620-625, 2011.

7. Sanyal A, Lajoie BR, Jain G and Dekker J: The long-range interaction landscape of gene promoters. Nature 489: 109-113, 2012.

8. Maurano MT, Humbert R, Rynes E, Thurman RE, Haugen E, Wang H, Reynolds AP, Sandstrom R, Qu H, Brody J, et al: Systematic localization of common disease-associated variation in regulatory DNA. Science 337: 1190-1195, 2012.

9. Smemo S, Tena JJ, Kim KH, Gamazon ER, Sakabe NJ, Gómez-Marín C, Aneas I, Credidio FL, Sobreira DR, Wasserman NF, et al: Obesity-associated variants within FTO form long-range functional connections with IRX3. Nature 507: 371-375, 2014.

10. Pasquali L, Gaulton KJ, Rodríguez-Seguí SA, Mularoni L, Miguel-Escalada I, Akerman I, Tena JJ, Morán I, Gómez-Marín C, van de Bunt M, et al: Pancreatic islet enhancer clusters enriched in type 2 diabetes risk-associated variants. Nat Genet 46: 136-143, 2014.

11. Kassem SA, Ariel I, Thornton PS, Hussain K, Smith V, Lindley KJ, Aynsley-Green A and Glaser B: p57(KIP2) expression in normal islet cells and in hyperinsulinism of infancy. Diabetes 50: 2763-2769, 2001.

12. Avrahami D, Li C, Yu M, Jiao Y, Zhang J, Naji A, Ziaie S, Glaser B and Kaestner KH: Targeting the cell cycle inhibitor p57Kip2 promotes adult human $\beta$ cell replication. J Clin Invest 124: 670-674, 2014.

13. Horike S, Mitsuya K, Meguro M, Kotobuki N, Kashiwagi A, Notsu T, Schulz TC, Shirayoshi Y and Oshimura M: Targeted disruption of the human LIT1 locus defines a putative imprinting control element playing an essential role in Beckwith-Wiedemann syndrome. Hum Mol Genet 9: 2075-2083, 2000.

14. Fitzpatrick GV, Soloway PD and Higgins MJ: Regional loss of imprinting and growth deficiency in mice with a targeted deletion of KvDMR1. Nat Genet 32: 426-431, 2002.

15. Arima $T$, Kamikihara $T$, Hayashida $T$, Kato $K$, Inoue $T$, Shirayoshi Y,Oshimura M,SoejimaH,Mukai T and WakeN:ZAC, LIT1 (KCNQ1OT1) and p57KIP2 (CDKN1C) are in an imprinted gene network that may play a role in Beckwith-Wiedemann syndrome. Nucleic Acids Res 33: 2650-2660, 2005.

16. Colsoul B, Schraenen A, Lemaire K, Quintens R, Van Lommel L, Segal A, Owsianik G, Talavera K, Voets T, Margolskee RF, et al: Loss of high-frequency glucose-induced $\mathrm{Ca}^{2+}$ oscillations in pancreatic islets correlates with impaired glucose tolerance in $\mathrm{Trpm}^{-/}$mice. Proc Natl Acad Sci USA 107: 5208-5213, 2010.

17. Brixel LR, Monteilh-Zoller MK, Ingenbrandt CS, Fleig A, Penner R, Enklaar T, Zabel BU and Prawitt D: TRPM5 regulates glucose-stimulated insulin secretion. Pflugers Arch 460: 69-76, 2010.

18. Kyriazis GA, Soundarapandian MM and Tyrberg B: Sweet taste receptor signaling in beta cells mediates fructose-induced potentiation of glucose-stimulated insulin secretion. Proc Natl Acad Sci USA 109: E524-E532, 2012.

19. Krishnan K, Ma Z, Björklund A and Islam MS: Role of transient receptor potential melastatin-like subtype 5 channel in insulin secretion from rat $\beta$-cells. Pancreas 43: 597-604, 2014.

20. Kawaguchi H, Asai A, Ohtsuka Y, Watanabe H, Wada T and Handa H: Purification of DNA-binding transcription factors by their selective adsorption on the affinity latex particles. Nucleic Acids Res 17: 6229-6240, 1989.

21. Inomata Y, Kawaguchi H, Hiramoto M, Wada T and Handa $H$ : Direct purification of multiple ATF/E4TF3 polypeptides from HeLa cell crude nuclear extracts using DNA affinity latex particles. Anal Biochem 206: 109-114, 1992. 
22. Wada T, Takagi T, Yamaguchi Y, Kawase H, Hiramoto M, Ferdous A, Takayama M, Lee KA, Hurst HC and Handa H: Copurification of casein kinase II with transcription factor ATF/E4TF3. Nucleic Acids Res 24: 876-884, 1996.

23. Nishio K, Masaike Y, Ikeda M, Narimatsu H, Gokon N, Tsubouchi S, Hatakeyama M, Sakamoto S, Hanyu N, Sandhu A, et al: Development of novel magnetic nano-carriers for high-performance affinity purification. Colloids Surf B Biointerfaces 64: 162-169, 2008.

24. Asfari M, Janjic D, Meda P, Li G, Halban PA and Wollheim CB: Establishment of 2-mercaptoethanol-dependent differentiated insulin-secreting cell lines. Endocrinology 130: 167-178, 1992.

25. Wada T, Watanabe H, Kawaguchi $\mathrm{H}$ and Handa $\mathrm{H}$ : DNA affinity chromatography. Methods Enzymol 254: 595-604, 1995.

26. Dignam JD, Lebovitz RM and Roeder RG: Accurate transcription initiation by RNA polymerase II in a soluble extract from isolated mammalian nuclei. Nucleic Acids Res 11: 1475-1489, 1983.
27. Shevchenko A, Wilm M, Vorm O and Mann M: Mass spectrometric sequencing of proteins silver-stained polyacrylamide gels. Anal Chem 68: 850-858, 1996.

28. Hiramoto M, Maekawa N, Kuge T, Ayabe F, Watanabe A, Masaike Y, Hatakeyama M, Handa H and Imai T: High-performance affinity chromatography method for identification of L-arginine interacting factors using magnetic nanobeads. Biomed Chromatogr 24: 606-612, 2010.

29. Bungartz G, Land H, Scadden DT and Emerson SG: NF-Y is necessary for hematopoietic stem cell proliferation and survival. Blood 119: 1380-1389, 2012.

30. Luo R, Klumpp SA, Finegold MJ and Maity SN: Inactivation of $\mathrm{CBF} / \mathrm{NF}-\mathrm{Y}$ in postnatal liver causes hepatocellular degeneration, lipid deposition, and endoplasmic reticulum stress. Sci Rep 1: 136,2011 